\section{Genomics education for Veterans Affairs nurses}

To the Editor: The authors of "Delivery of Clinical Genetic Consultative Services in the Veterans Health Administration" ${ }^{\prime \prime}$ and the invited commentary by Venne and Meyer $^{2}$ in the August 2014 issue highlight the need to educate Veterans Affairs (VA) providers on genetics knowledge and the availability of genetic services. The issue of genomic literacy surfaces at a critical time within the Veterans Health Administration. In 2006, then Secretary of Veterans Affairs R. James Nicholson initiated the VA's Genomic Medicine Program and established a Genomic Medicine Program Advisory Committee sponsored and managed by the Office of Research and Development. As part of the VA's Genomic Medicine Program, the Genomic Medicine Service continues to expand clinical genomic services. Under the research branch of the Genomic Medicine Program, the Million Veteran Program advances toward the goal of enrolling 1 million veteran volunteers. The Million Veteran Program will pave the way toward personalized medicine by creating a longitudinal cohort for genetic and health-related research. These developments reinforce the need for VA providers to understand genomics in the context of both research and clinical care. Specifically, we must identify patients who may benefit from the clinical guidance offered by genetic services and encourage participation in genomic research studies. Although physicians' genomics knowledge is integral to this discussion, the need for genomics education for other healthcare professionals cannot be understated.

Because nurses represent the nation's largest health-care profession and make up the Veterans Health Administration's largest clinical workforce, with more than 60,000 registered nurses, it seems timely to direct genomics education toward this discipline. Essential genetic and genomic competencies were established for all registered nurses and for those at the graduate level in 2009 and 2011, respectively, and have since been endorsed by professional nursing organizations. Nevertheless, many nurses have limited genomics knowledge because of the absence of readily available academic and continuing genomics education. Furthermore, there is a lack of academic faculty and clinicians with knowledge of and clinical expertise in genomics. ${ }^{3}$ Contributing to this knowledge and practice gap is the fact that demonstration of genomic competencies is not a visible component of mandatory evaluations of registered nurses' competency in clinical practice.

In 2011, the VA Office of Nursing Services and VA Office of Research and Development led a definitive effort toward improving patient care by developing genomics education targeted at the VA's nursing workforce. A panel of experts in the areas of nursing, genetics, and research created an online genomics education course and a genomic nursing competency assessment tool. Educational content for this effort was built from currently published competencies and recommendations from the national VA Genomics Nursing Competencies Workgroup, which identified 21 core genomic competencies for VA nurses. In June 2014, the course "Genomics in Your Nursing Practice" became available through the VA's online learning system to VA nurses nationwide, awarding 3.5 continuing education credits to those who successfully complete it.

As a broad overview, this online course reviews the following core elements of genomics: (i) the language of genomics; (ii) the ethical, legal, and social implications of genomic information; (iii) the nurse's role in collecting and documenting personal and family medical history; (iv) the nurse's role in applying genomics knowledge to assess risk; (v) the process for accessing the VA Genomic Medicine Service or other genetic services; and (vi) the key elements involved in follow-up from a genetics consultation across the continuum of care. Printable handouts, including a red flags family history checklist and patterns of inheritance cue card, are available within the course to facilitate translation of this knowledge to clinical care. An instrument to assess nurses' actual genomics knowledge, attitudes, and skills has been built into the education program using a pre-/posttest design. Results of this extensive educational effort will be published in the future.

Although the context of this discussion focuses on Veterans Health Administration Nursing Services, genomics education for nurses is now relevant in all academic and clinical practice settings, continuing education, and competency evaluations. The role of the nurse in genomics-based health care is a professional responsibility that should be endorsed by all health-care systems and encouraged by health-care leadership. Cross-cutting interprofessional educational efforts will help to ensure genomic literacy. Team-based approaches that value the demonstration of genomic competencies by all clinical disciplines offer the promise of improved and more effective personalized patient care. The combined efforts of the VA Office of Nursing Services and the VA Office of Research and Development aim to enhance these efforts.

At this time, "Genomics in Your Nursing Practice" is available only to VA health professionals through the VA Talent Management System. The course can be found by searching for the course title or the course number: 18527.

\section{DISCLOSURE}

The authors declare no conflict of interest. 
Kendra L. Schaa, ScM, CGCl, Gwen Anderson, $P h D, R N^{2}$, Anna C. Alt-White, $P h D, R N^{2}$, Christine E. Kasper, $P h D, R N^{2}$ and Andrea M. Boyd, PhD, $R N^{3}$

'Department of Veterans Affairs, Office of Research and Development, Washington, DC, USA; ${ }^{2}$ Department of Veterans Affairs, Office of Nursing Services, Washington, DC, USA; ${ }^{3}$ Department of Veterans Affairs, Wm. Jennings Bryan Dorn VA Medical Center, Columbia, South Carolina, USA. Correspondence: Kendra L. Schaa (Kendra.schaa@va.gov)

\section{REFERENCES}

1. Scheuner MT, Marshall N, Lanto A, et al. Delivery of clinical genetic consultative services in the Veterans Health Administration. Genet Med 2014;16:609-619.

2. Venne V, Meyer LJ. Genetics and the Veterans Health Administration. Genet Med 2014;16:573-575.

3. Calzone K, Jenkins J, Culp S, Caskey S, Badzek L. Introducing a new competency into nursing practice. J Nurs Regul 2014;5:40-47.

doi:10.1038/gim.2014.142 